\title{
Post traumatic parasomnia-case series
}

\begin{abstract}
Post traumatic stress disorders are associated with different sleep disorders such as insomnia, frequent awakenings, nightmares and periodic limb movement disorders. However parasomnias that are known to occur due to lack of REM atonia and different quality of post traumatic nightmares from idiopathic nightmares and dream enactment behavior disorder that is seen independent of an actual post traumatic stress disorder have received little attention. Three case series of post traumatic somnambulism, automatic texting and bruxism are reported to highlight this phenomenon.
\end{abstract}

Keywords: parasomnia, post traumatic stress disorders, REM atonia, somnambulism, bruxism
Volume I Issue 5 - 2017

\author{
Vyjayanthi S \\ Department of Psychiatry, MS Ramaiaha College, India
}

Correspondence: Vyjayanthi S, Department of Psychiatry, MS Ramaiaha College, Gokula Extension, Bangalore, 560054 Karnataka, India, Email vyjayanthi23@gmail.com

Received: November 16,2017 | Published: December 21, 2017
Abbrevations: PTSD, post traumatic stress disorders; REM, rapid eye movement

\section{Introduction}

Post traumatic stress disorders are associated with sleep disturbances that are considered as perhaps the core feature of the disorder. ${ }^{1}$ Nightmares, insomnia, hypersomnia, periodic limb movements have all been found to be associated with post traumatic stress disorders ${ }^{2}$ Non nightmare related awakenings have also been studied $^{3}$ Sleep disturbances can occur after a traumatic event without exhibiting all features of Post Traumatic Stress Disorders (PTSD). Sleep disturbances are found to predispose individuals to PTSD, hence considered as independent risk factors. ${ }^{1}$ Sleepwalking is found to occur in NREM stage4 sleep and is more purposive than periodic limb movement disorders. Overlapping of dissociation with sleep walking has been outlined in individual case reports ${ }^{3}$ Here patients have acted out or reenacted the trauma during stages of sleep and not while awake. ${ }^{4}$ Child sexual abuse has been a well established cause of post traumatic stress disorder and several forms of sleep disturbances. ${ }^{5}$ The following case series about parasomnias elucidate and support the above mentioned findings.

\section{Case vignette I: somnambulism or sleep walking}

A twenty two years old young man presented with complaints of sleep walking and moving out of his paying guest accommodation in the nights almost every night for several weeks and complete amnesia for these actions while awake. Patient was also noticed to carry and throw away electronic gadgets within his reach during these episodes including laptops, mobile phones. On further clarification it was noted that patient was working as a watchman in a hostel of college students. There was a desktop at his table to make entries of visitors. One morning he was summoned by the authorities and subjected to public shaming and accused of watching pornography on the desktop. Patient was unable to prove his innocence, though other students who were inmates of the hostel were using it after he retired to sleep. Patient was terminated from his job. Subsequently he was working at a motel as a cleaner. These episodes of sleep walking followed the traumatic event. Though patient had occasional day time episodes of palpitations, sweating and breathlessness, he had not sought help for the same. Patient received trauma focused psychotherapy with narrative therapy. He was also given lorazepam during the therapy and recovered completely in 6 months.

\section{Case vignette 2: automatic writing or texting in sleep}

A twenty three years old girl presented with complaints of automatic writing or texting to her mother at nights and no recall of the events during the day. Most of her text messages were about her mother being unjust in forgiving her biological brother who was 6 years older to her and had sexually abused her. Mother was a single parent widowed young was working as a school teacher. The incident had occurred when the girl was in third standard and the brother was in ninth standard. He had made her perform fellatio. At the time of presenting to the psychiatrist patient had suicidal thoughts, anhedonia, binge eating. Most of her texts in the night contained suicidal thoughts and anger towards her brother who was getting married? Patient responded well to narrative therapy, anger management and cognitive behavior therapy and pharmacotherapy for depression. Patient continued to be angry with her brother and disclosed the abuse to her sister-in law. Later patient married a person who was a citizen of another country and severed ties with her family of origin. However there were no episodes of nocturnal automatic writing in sleep and no recurrence of depression, her contact with the psychotherapist lasted for 4 years.

\section{Case vignette 3: bruxism or locked jaw}

A young girl aged 15 years presented with complaints of severe jaw movements ending in locked jaw every night for the past few months. Patient had received antipsychotics, anti-epileptics for the complaint and had not felt any relief. During a detailed interview it was revealed that patient had been bullied for wearing braces, being good at the local language of the state (This school had a law that children should not speak in the native language) teased about her stationary, and even for her extracurricular activities. Patient was bright and imaginative; she studied despite the daily ragging during which a teacher also joined in with other students in humiliating the child. Patient passed the entrance exams to join another school. However her attendance to the school was poor, since bruxism caused thinning of her condoyle and repeated locked jaws was painful. Mother would keep her awake at nights and sleep was viewed as unsafe behavior by both, the parent and child. When the child was 
extremely sleep deprived the movements did not occur when she slept. Patient was given baclofen and muscle relaxants for the spasm of masseters. Narrative exposure therapy to deal with the trauma of bullying was initiated. However, patient was exposed several types of body shaming at crucial junctures such as when she attained menarche and while she performed on stage. Treatment is in progress and her symptoms have reduced occurring intermittently. But patient has deferred attending the new school though she continues to enjoy reading science and creative writing.

\section{Discussion}

Studies on trauma have primarily focused on PTSD, with nightmares and insomnia as symptoms of PTSD. Trauma can trigger sleep disorders, including insomnia, nightmares, and parasomnias without the co morbid diagnosis of PTSD. ${ }^{4}$ Regarding parasomnias there are very few studies. However it is recognized that Post Traumatic Nightmares differ from idiopathic nightmares. Nightmare content in Post traumatic nightmares frequently relates to prior trauma and there are associated symptoms of panic attacks and there is dream enactment behavior in children and adults too. This is what was seen in the first case vignette. Patient was acting out in sleepwalking a reaction to the trauma experienced in reality. Patients are described to have REM without atonia, hence have dream enactment behaviors. The poly-somno-graphic studies have led to some researchers proposing a traumatic sleep disorder to be a separate nosology. ${ }^{4}$ The second case vignette also shows dream enactment behavior of automatic texting a dissociative disorder, here it occurred in sleep. The suicidal thoughts as expressed in these messages were introjected anger towards the brother.

Bruxism is a para-functional teeth grinding activity known to get triggered by stress and anxiety. $70 \%$ of bruxism is caused by stress and anxiety. ${ }^{6}$ Here it led to locked jaw and was symbolic of the bullying experienced by the child in school, as outlined in Forms of Bullying Scale body shaming of the child had a main focus on her jaws. ${ }^{7}$ In one of the reported studies on bruxism of the $38 \%$ of parents reporting that their children brux, familial history, open bedroom doors, drooling, sleep talking, and psychological disorders were significantly associated with the reported bruxism. Bullying by both teacher and fellow students led to a post traumatic school phobia, bruxism in sleep, day time sleepiness in the child. ${ }^{8}$

\section{Conclusion}

Post traumatic para-somnias need further research and attention. Rapid eye movement sleep without atonia show that post traumatic nightmares or dream enactment behaviors seen in survivors of civilian trauma need to be addressed carefully by the clinician and can have ameliorating effects on the post traumatic stress disorder, because disturbance of sleep is a core feature of the trauma.

\section{Acknowledgements}

None.

\section{Conflicts of interest}

The authors declared that there are no conflicts of interest.

\section{References}

1. Spoormaker VI, Montgomery P. Disturbed sleep in post-traumatic stress disorder: Secondary symptom or core feature? Sleep Med Rev. 2008;12(3):169-184.

2. Woodward SH, Leskin GA, Sheikh JI. Movement during sleep: associations with posttraumatic stress disorder, nightmares and co morbid panic disorder. Sleep. 2002;25(6):681-688.

3. Hartman D, Crisp AH, Sedgwick P, et al. Is there a dissociative process in sleepwalking and night terrors? Postgrad Med J. 2001;77:244-249.

4. Mysliwiec V, Brock MS, O'Reilly B, et al. Further evidence for a parasomnia incited by trauma. Sleep. 2016;39(12):2223-2224.

5. Sadeh A. Stress, Trauma, and Sleep in Children. Child and Adolescent Psychiatric Clinics of North America. 1996;5(3):685-700.

6. Cheifetz AT, Osganian SK, Allred EN, et al. Prevalence of bruxism and associated correlates in children as reported by parents. $J$ Dent Child (Chic). 2005;72(2):67-67.

7. Hutzell KL, Payne AA. The impact of bullying victimization on school avoidance. Youth Violence and Juvenile Justice. 2012;10(4):370-385.

8. Shaw T, Dooley JJ, Cross D, et al. The forms of bullying scale. (FBS): validity and reliability estimates for a measure of bullying victimization and perpetration in adolescence. Psychol Assess. 2013;25(4):1045-1057. 